\title{
Entanglement entropy and the Berry phase in the solid state
}

\author{
S. Ryu \\ Kavli Institute for Theoretical Physics, University of California, Santa Barbara, California 93106, USA
}

Y. Hatsugai

Department of Applied Physics, University of Tokyo, Hongo Bunkyo-ku, Tokyo 113-8656, Japan

(Received 11 January 2006; revised manuscript received 16 May 2006; published 26 June 2006)

\begin{abstract}
The entanglement entropy (von Neumann entropy) has been used to characterize the complexity of manybody ground states in strongly correlated systems. In this paper, we try to establish a connection between the lower bound of the von Neumann entropy and the Berry phase defined for quantum ground states. As an example, a family of translational invariant lattice free fermion systems with two bands separated by a finite gap is investigated. We argue that, for one-dimensional (1D) cases, when the Berry phase (Zak's phase) of the occupied band is equal to $\pi \times$ (odd integer) and when the ground state respects a discrete unitary particle-hole symmetry (chiral symmetry), the entanglement entropy in the thermodynamic limit is at least larger than $\ln 2$ (per boundary), i.e., the entanglement entropy that corresponds to a maximally entangled pair of two qubits. We also discuss how this lower bound is related to vanishing of the expectation value of a certain nonlocal operator which creates a kink in 1D systems.
\end{abstract}

DOI: 10.1103/PhysRevB.73.245115

PACS number(s): 71.10.Fd, 73.43.-f, 03.65.Ud, 77.22.Ej

\section{INTRODUCTION}

One of the most distinctive features of quantum phases of matter is that they are not completely characterized by their pattern of symmetry breaking (order parameters of some kind), which is in sharp contrast to classical statistical systems. Instead, quantum ground states should be described by their pattern of entanglement, such as topological or quantum order. ${ }^{1}$ However, beyond some simple textbook examples, e.g., a system of two coupled $S=1 / 2$ spins (qubits), we do not have many intuitions about quantum entanglement hidden in many-body wave functions. In the past few years, the entropy of entanglement (von Neumann entropy) ${ }^{2}$

$$
S_{A}=-\operatorname{tr}_{A} \rho_{A} \ln \rho_{A}, \quad \rho_{A}=\operatorname{tr}_{B}|\Psi\rangle\langle\Psi|,
$$

has been used to measure how closely entangled (or how "quantum") a given ground state wave function $|\Psi\rangle$ is. Here, the total system is divided into two subsystems $A$ and $B$ and $\rho_{A}$ is the reduced density matrix for the subsystem $A$ obtained by taking a partial trace over the subsystem $B$ of the total density matrix $\rho=|\Psi\rangle\langle\Psi|$. This quantity is zero for classical product states, whereas it takes a nontrivial value for valence-bond solid states (VBS), or resonating valence bond states (RVB) of quantum spin systems, say. Recently, the entanglement entropy at and close to quantum phase transitions in low-dimensional strongly correlated systems has been used as a new tool to investigate the nature of quantum criticality. ${ }^{3-9}$ Even though one can tell different quantum phases from the scaling of the entanglement entropy, it is still not completely understood what kind of information we can distill from the von Neumann entropy, other than that contained in conventional correlation functions.

On the other hand, a phase degree of freedom is also a specific feature of quantum mechanics. Indeed, Berry phases $^{10}$ associated with (many-body) wave functions in solid states are related to several interesting quantum phenomena which have no classical analogue. Probably, it is best epitomized by the Thouless-Kohmoto-Nightingale-Nijs (TKNN) formula in the integer quantum Hall effect (IQHE), ${ }^{11,12}$ in which gapped quantum phases are distinguished by an integral topological invariant originating from winding of the phase of wave functions. In addition to the IQHE, the Berry phase also appears in the King-SmithVanderbilt (KSV) formula ${ }^{13,14}$ of the theory of macroscopic polarization, its incarnation in quantum spin chains, ${ }^{15,16}$ and so on. An observable consequence of the nontrivial Berry phase is the existence of localized states at the boundaries when we terminate a system with boundaries. ${ }^{17-19}$

It is then tempting to ask if there is any connection between these two paradigms in quantum physics, namely entanglement and the Berry phase. In this paper, we discuss this issue by taking a family of translational invariant lattice free fermion systems in $d$ dimensions as an example. We bipartition the system into two subsystems $A$ and $B$ by introducing $(d-1)$-dimensional flat interfaces. Within this setup, we can reduce the calculation of the entropy to that in a one-dimensional system by the $(d-1)$-dimensional Fourier transform along the interface. We assume the existence of a finite energy gap $m$ above ground states which is inversely proportional to the correlation length, $m \sim \xi_{\text {corr }}^{1}$ (when measured in the unit of the bandwidth). Furthermore, for simplicity, we consider the case in which there are only two bands that are separated by a gap.

In this paper, we consider the Berry phase associated with a response of a quantum ground state to a continuous twist of the boundary condition. For the case of free lattice fermion systems, for which a ground state is given by a filled FermiDirac sea, this Berry phase is a phase acquired by an adiabatic transport of the Bloch wave functions in the momentum space, also called Zak's phase. ${ }^{20}$ Physically, it is related to macroscopic polarization of the Fermi-Dirac sea. ${ }^{13}$ The beauty of the simple two-band example that we discuss is that the Berry phase for the quantum ground state can be easily computed and visualized, following the pioneering 


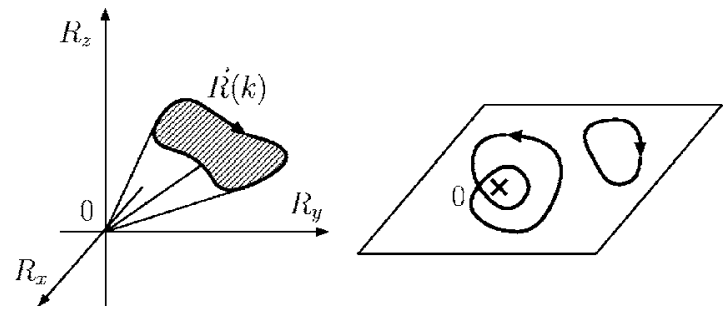

FIG. 1. (Left) The loop defined by a three-component vector $\boldsymbol{R}(k)$ associated with the Hamiltonian in momentum space [Eq. (2.1)]. (Right) Loops for chiral symmetric Hamiltonians.

work by Berry ${ }^{10}$ (see Sec. II and Fig. 1 below).

With these setups, we will demonstrate that taking the partial trace over a subsystem corresponds to creating boundaries in a system. Two contributions to the entanglement entropy will then be identified. The first one is of a type already discussed in a flurry of recent works focusing on detection of quantum critical points. This contribution to the entanglement entropy is largely controlled by the correlation length $\xi_{\text {corr }}$. For example, in one-dimensional (1D) many-body systems close to criticality, the entanglement entropy obeys a logarithmic law $S_{A} \sim \mathcal{A}(c / 6) \ln \xi_{\text {corr }} / a$, where $c$ is the central charge of the conformal field theory that governs the criticality, $a$ is the lattice constant, and $\mathcal{A}$ is the number of boundary points of $A .^{6,8}$

On the other hand, the second contribution to the entropy comes from the localized boundary states of the correlation matrix that exist when the Berry phase of the ground-state wave function is nonvanishing. Especially, when the Berry phase is equal to $\pi \times$ (odd integer) and when the ground state respects discrete symmetries of some sort, the localized boundary states are topologically protected as discussed in Refs. 17 and 18. For this case, we will show that the contribution from the boundary states to the von Neumann entropy is $\ln 2$ per boundary, i.e., the same amount of entropy carried by a maximally entangled pair of two qubits. We will also illustrate, by taking a specific limit, that when $\gamma \neq 0$, the von Neumann entropy from the boundary states is that of partially entangled qubits.

We also discuss that the $\ln 2$ contribution to the von Neumann entropy is related to vanishing of the expectation value of a certain nonlocal operator which creates a kink in 1D systems. This connection between the entanglement entropy and the kink operator is, in flavor, similar to discussions in Refs. 8 and 44 in which the entanglement entropy is expressed as the expectation values of twist operators in conformal field theories.

The rest of the paper is organized as follows. In Sec. II, we start our discussions with 1D translational invariant Hamiltonians with two bands separated by a finite gap. The Berry phase is introduced as an expectation value of a specific nonlocal operator that twists the phase of the wave functions. We then discuss its connection to the entanglement entropy by making use of the correlation matrix. The calculation of the entanglement entropy is, in general, a rather difficult task at least analytically. Furthermore, the Berry phase contribution to the entropy might not be of a perturbative nature. We thus consider two limiting situations. In Sec.
II B, we take the limit of the small correlation length $\xi_{\text {corr }}$ $\ll 1$ and zero bandwidth. In this specific limit, we can express the entanglement entropy as a function of the Berry phase $\gamma$. We next focus on cases with a discrete unitary particle-hole symmetry (chiral symmetry) in Sec. II C. Except for requiring the chiral symmetry, any parameters of the Hamiltonian (the band structure) can be arbitrary. Once we impose the chiral symmetry, the Berry phase $\gamma$ can take only discrete values, integral multiple of $\pi$. We then show when $\gamma=\pi \times$ (odd integer), the entanglement entropy is bounded below as $S_{A} \geqslant 2 \ln 2$. In Sec. III, we relate the lower bound of the entropy at $\gamma=\pi \times$ (odd integer) to the vanishing of a nonlocal operator that creates a kink. In Sec. IV, these discussions are applied to a higher-dimensional example, a 2D superconductor with nonzero TKNN integer. We conclude in Sec. V.

\section{1D TWO-BAND SYSTEMS}

We start from the following 1D translational invariant Hamiltonians with two bands separated by a finite gap,

$$
\mathcal{H}=\sum_{x, x^{\prime}}^{\mathrm{PBC}} \boldsymbol{c}_{x}^{\dagger} H_{x-x^{\prime}} \boldsymbol{c}_{x^{\prime}}, \quad H_{x-x^{\prime}}=\left(\begin{array}{cc}
t_{+} & \Delta \\
\Delta^{\prime} & t_{-}
\end{array}\right)_{x-x^{\prime}} .
$$

Here, a pair of fermion annihilation operators $\boldsymbol{c}_{x}^{\mathrm{T}}=\left(c_{+}, c_{-}\right)_{x}$ is assigned for each cite, $x, x^{\prime}=1, \ldots, N$, and the hermiticity of $\mathcal{H}$ implies $t_{\iota, x-x^{\prime}}=t_{\iota, x^{\prime}-x}^{*}$ and $\Delta_{x-x^{\prime}}=\left(\Delta_{x^{\prime}-x}^{\prime}\right)^{*}$ for $\iota= \pm$. We impose the periodic boundary condition (PBC) on the 1D lattice. In spite of its simplicity, this Hamiltonian (2.1) has a wide range of applicability, such as the Bogoliubov-de Gennes Hamiltonian in superconductivity, graphite systems, ${ }^{17}$ ferroelectricity of organic materials and perovskite oxides, ${ }^{21}$ and the slave boson mean-field theory for spin liquid states.

By the Fourier transformation $\boldsymbol{c}_{x}=N^{-1 / 2} \Sigma_{k \in \mathrm{BZ}} e^{i k x} \boldsymbol{c}_{k}$, where the summation over $k$ extends over the first Brillouin zone (BZ), $k=2 \pi n / N(n=1, \ldots, N)$, the Hamiltonian in the momentum space is given by $\mathcal{H}=\sum_{k \in \mathrm{BZ}} \boldsymbol{c}_{k}^{\dagger} H(k) \boldsymbol{c}_{k}$, with $H(k)$ $:=\sum_{x} e^{-i k x} H(x)$. If we introduce an "off-shell" four-vector $R^{\mu=0,1,2,3}(k) \in \mathbb{R} \quad$ by $\quad R^{0}(k) \mp R^{3}(k):=t_{ \pm}(k), \quad-R^{1}(k)+\mathrm{i} R^{2}(k)$ $:=\Delta(k)$, we can rewrite the Hamiltonian in the momentum space as

$$
\mathcal{H}=\sum_{k \in \mathrm{BZ}} \boldsymbol{c}_{k}^{\dagger} R^{\mu}(k) \sigma_{\mu} \boldsymbol{c}_{k},
$$

where $\boldsymbol{\sigma}_{\mu}=\left(\sigma_{0},-\boldsymbol{\sigma}\right)$ with $\sigma_{0}=\mathbb{I}_{2}$.

Observing that $R^{\mu} \sigma_{\mu}$ is diagonalized by the same eigenvectors as those of $R_{i} \sigma_{i}=\boldsymbol{R} \cdot \boldsymbol{\sigma}$ (but with different eigenvalues), normalized eigenstates $\vec{v}_{ \pm}$for $R^{\mu} \sigma_{\mu}$ are given by, when $\boldsymbol{R}$ is not in the Dirac string, $\left(R^{1}, R^{2}\right) \neq(0,0),{ }^{10}$

$$
\vec{v}_{ \pm}=\frac{1}{\sqrt{2 R\left(R \mp R^{3}\right)}}\left(\begin{array}{c}
R^{1}-i R^{2} \\
\pm R-R^{3}
\end{array}\right),
$$

where $R=|\boldsymbol{R}|$ (it should not be confused with $R^{0}$ ), and the eigenvalue for $\vec{v}_{ \pm}$is $E_{ \pm}=R^{0} \mp R$. The Hamiltonian is then diagonalized as $\mathcal{H}=\sum_{k} \boldsymbol{\alpha}_{k}^{\dagger} \operatorname{diag}\left(E_{+}, E_{-}\right)_{k} \boldsymbol{\alpha}_{k}$, where $c_{\iota, k}$ 
$=\left(\vec{v}_{\sigma}\right)^{\iota} \alpha_{\sigma, k}$. As we assume there is a finite gap for the entire Brillouin zone, $E_{+}>E_{-},{ }^{\forall} k \in \mathrm{BZ}$. The vacuum $|\Psi\rangle$ is the filled Fermi sea $|\Psi\rangle=\prod_{k \in \mathrm{BZ}} \alpha_{-, k}^{\dagger}|0\rangle$.

The Berry phase can be defined through the expectation value of the twist operator,

$$
z:=\exp \left[i \frac{2 \pi}{N} \sum_{x} x n_{x}\right],
$$

where $n_{x}$ is the electron number operator at site $x, n_{x}$ $=\Sigma_{\iota} c_{x, \iota}^{\dagger} c_{x, \iota}$. This operator twists the phase of wave functions along the $x$ direction over the wide length scale, $N$. If we use the $S_{z}$ component of a spin operator, say, instead of $n_{x}$, we can define the twist operator in spin systems in a similar fashion. The twist operator has been used to characterize low-dimensional quantum systems ${ }^{16}$ and to describe macroscopic polarization of insulators, ${ }^{13}$ say.

For the Fermi sea $|\Psi\rangle$, the expectation value of the twist operator is calculated as

$$
\langle\Psi|z| \Psi\rangle=(-1)^{N+1} \exp \left[i \gamma-\xi_{\text {loc }}^{2} / N+O\left(1 / N^{2}\right)\right],
$$

where the Berry phase (Zak's phase) $\gamma$ is given by a line integral of the gauge field $A(k)$ over the 1D BZ, ${ }^{13,14,20,22}$

$$
\begin{gathered}
i A_{x}(k):=\left\langle v_{-}(k)\left|\frac{d}{d k}\right| v_{-}(k)\right\rangle, \\
\gamma:=i \int_{0}^{2 \pi} i A_{x}(k) d k .
\end{gathered}
$$

For the Fermi sea $|\Psi\rangle$ derived from the Hamiltonian (2.2), $\gamma$ is simply equal to half of the solid angle sustained by a loop defined by $\boldsymbol{R}(k)$ in $\boldsymbol{R}$ space (see Fig. 1). ${ }^{10,17}$. On the other hand, the $O(1 / N)$ correction to $\ln \langle\Psi|z| \Psi\rangle$ is real and given by the integral of the quantum metric $g_{x x}(k)$ over the $\mathrm{BZ},{ }^{23}$

$$
\begin{gathered}
g_{x x}(k):=\operatorname{Re}\left\langle\partial_{k} v_{-} \mid \partial_{k} v_{-}\right\rangle-\left\langle\partial_{k} v_{-} \mid v_{-}\right\rangle\left\langle v_{-} \mid \partial_{k} v_{-}\right\rangle, \\
\xi_{\text {loc }}^{2}:=\pi \int_{0}^{2 \pi} g_{x x}(k) d k .
\end{gathered}
$$

The localization length $\xi_{\text {loc }}$ plays a similar role to $\xi_{\text {corr }}$ and is known to be related to most localized Wannier states in an insulating phase. ${ }^{23}$

\section{A. Truncated correlation matrix and its zero modes}

We next partition the system into two parts, $A=\{x \mid x$ $\left.=1, \ldots, N_{A}\right\}$ and $B=\left\{x \mid x=N_{A}+1, \ldots, N_{B}\right\}$ with $N_{A}+N_{B}=N$, and ask, with the von Neumann entropy $S_{A}$, to what extent these two subsystems are entangled. Instead of directly tracing out the subsystem $B$ following the definition (1.1), we can make use of correlation matrix $C_{\iota \lambda}(x-y):=\left\langle c_{(x, \iota)}^{\dagger} c_{(y, \lambda)}\right\rangle$ as shown in Ref. 24. From the entire correlation matrix, we extract the submatrix $\left\{C_{\iota \lambda}(x-y)\right\}_{x, y \in A}$, where $x$ and $y$ are restricted in the subsystem $A$. The entanglement entropy is then given by

$$
S_{A}=-\sum_{a}\left[\zeta_{a} \ln \zeta_{a}+\left(1-\zeta_{a}\right) \ln \left(1-\zeta_{a}\right)\right]
$$

where $\zeta_{a}$ are the eigenvalues of the truncated correlation ma$\operatorname{trix}\left\{C_{\iota \lambda}(x-y)\right\}_{x, y \in A}$.

With the whole set of the eigenvalues $\left\{E_{ \pm}(k)\right\}$ and eigen wave functions $\left\{v_{ \pm}(\boldsymbol{k})\right\}$ [Eq. (2.3)] in hand, the correlation matrix $C_{\iota \lambda}(x-y)=N^{-1} \sum_{k \in \mathrm{BZ}} \mathrm{e}^{-i k(x-y)} C_{\iota \lambda}(k)$ is calculated exactly as

$$
C_{\iota \lambda}(k)=\frac{1}{2}\left[n^{\mu}(k) \sigma_{\mu}\right]_{\iota \lambda},
$$

where we have introduced an "on-shell" four-vector $n^{\mu}$ by $n^{\mu}=(1, \boldsymbol{R} / R)$. It should be noted that a set of Hamiltonians can share the same ground-state wave function and thus the same correlation matrix.

The basic idea we will use to discuss the entanglement entropy is to think that the correlation matrix $C(x-y)$ defines a 1D "Hamiltonian" with PBC. This "Hamiltonian" (let us call it the correlation matrix Hamiltonian or the $\mathcal{C}$ Hamiltonian for simplicity) has the same set of eigen wave functions as the original Hamiltonian but all the eigenvalues are given by either 1 or 0 . The range of hopping elements in the generated system is of order of the inverse gap of the original Hamiltonian. That is if there is a finite gap, the $\mathcal{C}$ Hamiltonian is local (short-ranged).

Now, all we need to know is what energy spectrum the $\mathcal{C}$ Hamiltonian will have when we cut it into two parts, defined by $A$ and $B$. This is the same question asked in Ref. 17, in which a criterion to determine the existence of zero-energy edge states is presented. There are two types of eigenvalues in the energy spectrum of the truncated $\mathcal{C}$ Hamiltonian in the thermodynamic limit $N_{A} \rightarrow \infty$. Eigenvalues of the first type are identical to their counterpart in the periodic (untruncated) system. On top of it, there appear localized boundary states whose eigenvalues are located within the bulk energy gap. Since the eigenvalues that belong to the bulk part of the spectrum are either 1 or 0 , they do not contribute to the entanglement entropy as seen from Eq. (2.8), whereas the boundary modes do.

The question is then how many boundary states appear and with what energy when the system is truncated. As suggested from the KSV formula in macroscopic polarization, the nonvanishing Berry phase of the filled band of the $\mathcal{C}$ Hamiltonian implies the existence of states localized near the boundary. Here, note that the Berry phase for the generated system ( $\mathcal{C}$ Hamiltonian) is identical to that of the original system, since the original and generated Hamiltonians share the same set of eigen wave functions.

\section{B. Dimerized limit}

To know the number of localized states that appear in the spectrum and the energy eigenvalues thereof is, in general, a difficult task. In this subsection, we consider a limiting situation in which the localization length in Eq. (2.7) is small, $\xi_{\text {corr }} \ll 1$, and the bandwidth of the energy spectrum is zero. More precisely, let us consider the case in which the correlation matrix is given by a four-vector $n^{\mu}[\mathrm{Eq}$. (2.9)] with 


$$
\boldsymbol{R}(k)=(-\Delta \cos k,-\Delta \sin k, \xi),
$$

where $\Delta, \xi \in \mathbb{R}$. There is a family of Hamiltonians having this correlation matrix which includes the following "dimerized" Hamiltonian:

$$
\mathcal{H}=\sum_{x}\left[\sum_{\iota} \iota \xi c_{x \iota}^{\dagger} c_{x \iota}+\Delta c_{x+1,+}^{\dagger} c_{x,-}+\text { H.c. }\right] \text {. }
$$

The inverse Fourier transformation of Eq. (2.10) gives the correlation matrix in the tight-binding notation,

$$
\mathcal{C}=\sum_{x}\left[\sum_{\iota} \frac{(R-\iota \xi)}{2 R} c_{x \iota}^{\dagger} c_{x \iota}-\frac{\Delta}{2 R} c_{x+1,+}^{\dagger} c_{x,-}+\text { H.c. }\right] .
$$

This $\mathcal{C}$ Hamiltonian can be diagonalized for both periodic and truncated boundary conditions by introducing the "dimer" operators via $d_{ \pm, x+(1 / 2)}^{\dagger}=\left(c_{x,+}^{\dagger} \pm c_{x+1,-}^{\dagger}\right) / \sqrt{2}$ (see also Appendix A). The truncated $\mathcal{C}$ Hamiltonian has $(N-1)$-fold degenerate eigenvalues $\zeta=0,1$, and two eigenvalues $\zeta$ $=\left(1 \pm \frac{\xi}{R}\right) / 2$ that correspond to edge states. The entanglement entropy (in the thermodynamic limit) is then computed as

$$
\frac{1}{2} S_{A}=-\frac{\gamma}{2 \pi} \ln \frac{\gamma}{2 \pi}-\frac{(2 \pi-\gamma)}{2 \pi} \ln \frac{2 \pi-\gamma}{2 \pi},
$$

where the Berry phase $\gamma$ for the correlation matrix (2.10) is $\gamma / \pi=1-\xi / R$. In the two extreme cases, $\xi=0$ and $\xi \rightarrow \pm \infty$, we have $S_{A}(\xi=0)=2 \ln 2$ and $S_{A}(\xi \rightarrow \pm \infty)=0$, respectively. The entanglement entropy in the present case is a convex function with respect to $\gamma \in[0,2 \pi]$ and the maximum is achieved when $\gamma=\pi$, whereas two minima are located at $\gamma$ $=0,2 \pi$.

\section{Case of $\gamma=\pi$ with chiral symmetry}

Although the formula (2.13) clearly shows the relation between the Berry phase and the entanglement entropy in a specific limit, it is rather difficult to extend Eq. (2.13) to more generic situations. However, if we impose a discrete symmetry implemented by a unitary particle-hole transformation, so-called chiral symmetry, on the $\mathcal{C}$ Hamiltonian, it is possible to make a precise prediction for the number of boundary states that has an eigenvalue $\zeta=1 / 2$, following the same line of discussions in Ref. 17.

When the system respects the chiral symmetry, we can find a unitary matrix that anticommutes with the one-particle Hamiltonian. For this case, $\boldsymbol{n}(k)$ is restricted to lie on a plane cutting the origin in $\boldsymbol{R}$ space, which in turn means that the Berry phase for the lower band of $\mathcal{H}$ is equal to $n \pi(n$ $\in \mathbb{N}$ ) (see Fig. 1).

When $n$ is odd, we can show that there are at least a pair of boundary modes at $\zeta=1 / 2$, one of which is localized at the left end and the other at the right. ${ }^{25}$ [The system with $\gamma= \pm \pi$ is, in a sense, "dual" to that with the vanishing Berry phase where there is no boundary state (see Appendix A)]. Basically, this is because, when $n$ is odd, it is always possible to deform the $\mathcal{C}$ Hamiltonian into a "reference" one without closing the bulk energy gap and without changing the Berry phase. The reference $\mathcal{C}$ Hamiltonian is similar to the dimerized example (2.12) for which one can exactly show the existence of $n$ pairs of edge modes at $\zeta=1 / 2$. In the course of deformation, the edge modes present in the reference $\mathcal{C}$ Hamiltonian can move away from $\zeta=1 / 2$. However, due to the chiral symmetry, the edge modes can escape from $\zeta$ $=1 / 2$ only in a pairwise fashion, i.e., an edge state localized on the left/right must always be accompanied by the one localized on the same end and with the opposite eigenvalue with respect to $\zeta=1 / 2$. When $n$ is odd, a pair of edge modes (one for each end) cannot have its partner and hence we are left with at least one edge mode per boundary located exactly at $\zeta=1 / 2$. See Ref. 17 for more detailed discussions.

Then, the lower bound of the entanglement entropy is given by

$$
S_{A} \geqslant-\ln \frac{1}{2}-\ln \frac{1}{2}=2 \ln 2 .
$$

This lower bound is equal to the entanglement entropy contained in a dimer for each end of the original model, which is consistent with the fact that the origin of the boundary states discussed above can be traced back to dimers in the reference Hamiltonian to which a given target Hamiltonian is adiabatically connected.

There can be other contributions from boundary states that are not connected to a dimer in the above sense. Indeed, as we will explicitly demonstrate below, these kinds of boundary modes proliferate as we approach a quantum critical point whose number grows as $\sim \mathcal{A}(c / 6) \ln \xi_{\text {corr }} / a$, and finally gives rise to the logarithmic divergences at the critical point. ${ }^{8}$

Note also that our discussion here does not apply gapless systems since the matrix elements of the $\mathcal{C}$ Hamiltonian are long-ranged in this case.

\section{Example: Su-Schrieffer-Heeger model}

As an example, let us look at a situation in which two phases with the Berry phase $\gamma=\pi$ and 0 are connected by a quantum phase transition point. Physically, such an example is provided by the Su-Schrieffer-Heeger (SSH) model for a chain of polyacetylene. The 1D tight-binding Hamiltonian for the SSH model for a chain of polyacetylene is given by $\mathcal{H}=\sum_{i=1}^{N_{i}} t\left[-1+(-1)^{i} \phi_{i}\right]\left(c_{i}^{\dagger} c_{i+1}+\right.$ H.c. $),{ }^{26}$ where $\phi_{i}$ represents dimerization at the $i$ th site, and an alternating sign of the hopping elements reflects dimerization between the carbon atoms in the molecule. Here, we treat the lattice in a classical fashion and neglected its elastic (kinetic) energy. Taking $\phi_{i}$ $=\phi=$ const, $t=1$, and defining a spinor at $x=2 i-1$ by $\boldsymbol{c}_{x}$ $=\left(c_{i}, c_{i+1}\right)^{\mathrm{T}}$, the Hamiltonian can be written as $\left(N=N_{i} / 2\right)$

$$
\begin{aligned}
\mathcal{H}= & \sum_{x=1}^{N} \boldsymbol{c}_{x}^{\dagger}\left(-(1+\phi)^{-(1+\phi)}\right) \boldsymbol{c}_{x}-\boldsymbol{c}_{x}^{\dagger}\left(\begin{array}{ll}
0 \\
1-\phi
\end{array}\right) \boldsymbol{c}_{x+1} \\
& + \text { H.c. }
\end{aligned}
$$

Under the PBC, the SSH Hamiltonian can be diagonalized as Eq. (2.2) with $R_{x}(k)=-1-\phi-(1-\phi) \cos k, \quad R_{y}(k)=(-1$ $+\phi) \sin k, R_{z}(k)=0$. 

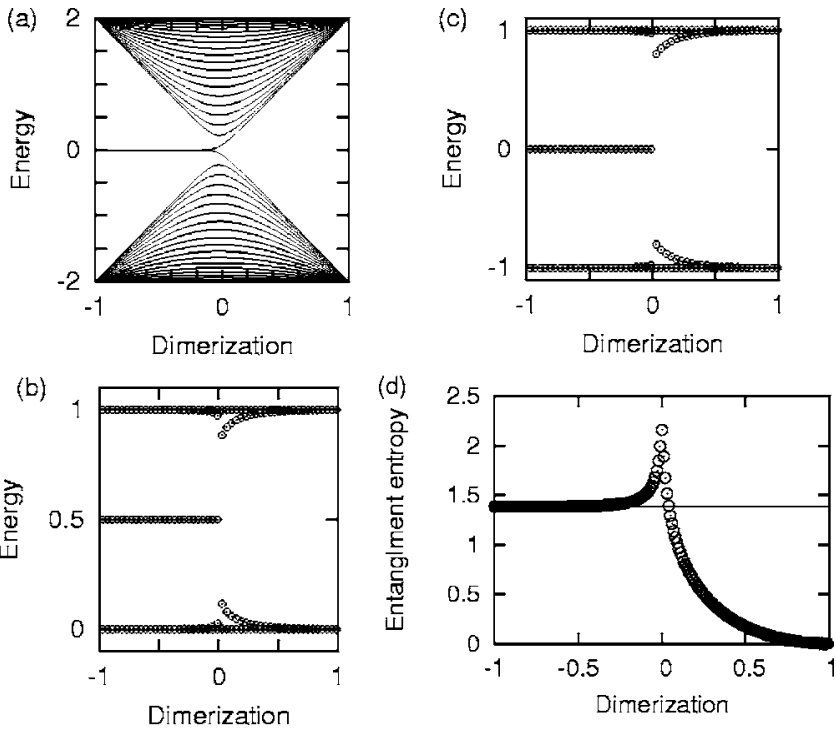

FIG. 2. The energy spectra of (a) the Hamiltonian $\mathcal{H}$ with open ends, (b) the truncated correlation matrix $\mathcal{C}$, and (c) the matrix $\mathcal{S}$ (see Sec. III) as a function of the dimerization parameter $\phi \in[-1,1]$ for the SSH model. Both energy and dimerization are measured in units of the hopping amplitude, $t$. (d) The entanglement entropy of the SSH model.

For $\phi \in[-1,0)$, the Berry phase is given by $\gamma=\pi$, whereas for $\phi \in(0,1], \gamma=0$. These two phases are separated by a quantum phase transition at $\phi=0$. Following the discussion in Ref. 17, there is at least a pair of boundary states for $\phi \in[-1,0)$ when we terminate the system. Indeed, for the numerically computed energy spectrum of the SSH model with open ends [Fig. 2(a)] for $\phi \in[-1,+1]$, there is a pair of edge states in the bulk energy gap when $\phi \in[-1,0)$.

The entanglement entropy is calculated by diagonalizing the $\mathcal{C}$ Hamiltonian. The energy spectrum of the $\mathcal{C}$ Hamiltonian with open ends is shown in Fig. 2(b). Again, there is a pair of boundary states for $\phi \in[-1,0)$, and for this case, $S_{A}$ is bounded from below as $S_{A} \geqslant 2 \ln 2$ [Fig. 2(d)]. When we approach the transition point $\phi=0$, some bulk eigenvalues turn into the boundary eigenvalues and they give rise to extra contributions other than the zero-energy boundary states. Similar behavior of the entanglement entropy is discussed for the quantum Ising chain in transverse magnetic field, where the $2 \ln 2$ entropy originates from a Schrödinger cat state composed of all spin-up and -down configurations.

\section{CONNECTION TO A KINK OPERATOR}

We have seen that bipartitioning the system corresponds to an introduction of a sharp "boundary" (interface). In this section, we will realize it by a nonlocal operator, a kink operator

$$
\eta:=\exp \left[i \sum_{x} \varphi(x) n_{x}\right], \quad \eta^{\dagger}=\eta^{-1}
$$

where

$$
\varphi(x):= \begin{cases}0, & x \in A, \\ \pi, & x \in B .\end{cases}
$$

The geometric mean of this kink operator is the twist operator. $^{27}$

The kink operator attaches a phase factor $\varphi(x)$ for the fermion operators at site $x$,

$$
\eta^{\dagger} c_{x \iota} \eta=e^{+i \varphi(x)} c_{x \iota}, \quad \eta^{\dagger} c_{x \iota}^{\dagger} \eta=e^{-i \varphi(x)} c_{x \iota}^{\dagger}
$$

Thus, if we introduce the reduced density operator through

$$
\tilde{\rho}_{A}:=\frac{1}{2}\left[\eta|\Psi\rangle\left\langle\Psi\left|\eta^{\dagger}+\right| \Psi\right\rangle\langle\Psi|\right],
$$

the matrix elements $\operatorname{tr}\left[c_{x, \iota}^{\dagger} c_{y, \lambda} \tilde{\rho}_{A}\right]$ are vanishing whenever $x$ $\in A$ and $y \in B$ and vice versa, whereas they coincide with the correlation matrix $C_{\iota \lambda}(x-y)$ when $x, y \in A$. Unlike $\rho_{A}$, the matrix elements $\operatorname{tr}\left[c_{x, \iota}^{\dagger} c_{y, \lambda} \widetilde{\rho}_{A}\right]$ are nonzero even for the $B$ subsystem. This "padding" does nothing, however.

In the following, we will discuss the expectation value of the kink operator $\langle\Psi|\eta| \Psi\rangle$ with respect to a given ground state wave function $|\Psi\rangle$ which is related to the expectation value of $\tilde{\rho}_{A}$ as $\left\langle\Psi\left|\tilde{\rho}_{A}\right| \Psi\right\rangle=\frac{1}{2}\left[|\langle\Psi|\eta| \Psi\rangle|^{2}+1\right]$. As we will see, the vanishing of $\langle\Psi|\eta| \Psi\rangle$ is closely related to a $\ln 2$ contribution to $S_{A}$ discussed in the previous section. This can be understood intuitively as follows. Classical wave functions can be written as a product state and are rather insensitive to the kink operator. Thus, the ground state with the kink operator inserted $\eta|\Psi\rangle$ has a large overlap with the original ground state $|\Psi\rangle$. On the other hand, the kink operator destroys dimers if the Berry phase of the ground state is $\pi$ $\times$ (odd integer). As a consequence, the overlap $\langle\Psi|\eta| \Psi\rangle$ is very small in this quantum phase, which in turn suggests that quasiparticles that constitute the continuum spectrum above the ground state can be interpreted as a kink created by $\eta$. Thus the kink operator is capable of distinguishing the quantum phases with different entanglement properties.

To put the above statement in a quantum information perspective, remember the reduced density matrix $\widetilde{\rho}_{A}$ is in general in a mixed state,

$$
\tilde{\rho}_{A}=\sum_{n} p_{n}\left|\Psi_{n} \otimes 0\right\rangle\left\langle\Psi_{n} \otimes 0\right|,
$$

where $\left|\Psi_{n}\right\rangle$ belongs to the subsystem $A$, and $\Sigma_{n} p_{n}=1$. When the wave function $|\Psi\rangle$ happens to be a completely entanglement-free product state, $|\Psi\rangle=\left|\Psi_{A}\right\rangle \otimes\left|\Psi_{B}\right\rangle$, the reduced density matrix $\tilde{\rho}_{A}$ is in a pure state, i.e., $p_{n \neq 1}=0, p_{1}$ $=1,\left|\Psi_{1}\right\rangle=\left|\Psi_{A}\right\rangle$. On the other hand, when $|\Psi\rangle$ is highly entangled, taking partial trace over the $B$ subsystem generates many pure states $\left|\Psi_{n}\right\rangle$ with nonzero weight $0<p_{n}<1$. How far a given state $\left|\Psi_{n}\right\rangle$ is from a product state can then be measured by taking the expectation value of the reduced density matrix $\widetilde{\rho}_{A}$,

$$
\left\langle\Psi\left|\widetilde{\rho}_{A}\right| \Psi\right\rangle=\sum_{n} p_{n}\left\langle\Psi \mid \Psi_{n} \otimes 0\right\rangle\left\langle\Psi_{n} \otimes 0 \mid \Psi\right\rangle .
$$

Clearly, it is equal to 1 when $\left|\Psi_{n}\right\rangle$ is a product state whereas it is expected to be less than 1 for entangled states. 
In the following subsections, we will establish that in an insulating phase the expectation value of the kink operator is zero in the thermodynamic limit when the Berry phase is $\pi \times$ (odd integer), whereas it is finite otherwise.

\section{A. Expectation value of the kink operator as a determinant}

The computation of the expectation value of the kink operator for a Fermi-Dirac sea $|\Psi\rangle=\Pi_{k \in \mathrm{BZ}} \alpha_{-, k}^{\dagger}|0\rangle$ goes as follows. In the momentum space, the phase attachment transformation (3.3) reads

$$
\eta^{\dagger} \boldsymbol{c}_{k} \eta=\sum_{q} f_{q} \boldsymbol{c}_{k-q}, \quad \eta^{\dagger} \boldsymbol{c}_{k}^{\dagger} \eta=\sum_{q} f_{q}^{*} \boldsymbol{c}_{k-q}^{\dagger},
$$

where we introduced the Fourier components of $e^{i \varphi(x)}$ by

$$
e^{i \varphi(x)}=f(x)=\sum_{q \in \mathrm{BZ}} f_{q} e^{i q x},
$$

with $q=2 \pi n_{q} / N\left(n_{q} \in \mathbb{N}\right)$ and

$$
f_{q}=2 \frac{1-e^{-i \pi n_{q}}}{1-e^{-i 2 \pi n_{q} / N}}= \begin{cases}\frac{4}{1-e^{-i 2 \pi n_{q} / N}}, & n_{q}=1,3, \ldots, N-1, \\ 0, & n_{q}=0,2, \ldots, N-2 .\end{cases}
$$

In a basis that diagonalizes the Hamiltonian,

$$
\eta^{\dagger} \boldsymbol{\alpha}_{k} \eta=\sum_{k^{\prime}} \boldsymbol{S}_{k, k^{\prime}}^{\dagger} \boldsymbol{\alpha}_{k^{\prime}}, \quad \eta^{\dagger} \boldsymbol{\alpha}_{k}^{\dagger} \eta=\sum_{k^{\prime}} \boldsymbol{\alpha}_{k^{\prime}}^{\dagger} \boldsymbol{S}_{k, k^{\prime}},
$$

where a $2 N \times 2 N$ matrix $S_{(k \iota)\left(k^{\prime} \lambda\right)}$ is given by

$$
\boldsymbol{S}_{(k \iota)\left(k^{\prime} \lambda\right)}=\sum_{q} f_{q}^{*}\left[v^{\dagger}(k-q) v(k)\right]_{\iota \lambda} \delta_{k-q, k^{\prime}},
$$

and $v^{\dagger}(p)=\left(v_{+}^{\dagger}(p), v_{-}^{\dagger}(p)\right)$.

The expectation value of the kink operator with respect to $|\Psi\rangle$ is then represented as the determinant of $N \times N$ matrix $S_{(k-)\left(k^{\prime}-\right)}$,

$$
\langle\Psi|\eta| \Psi\rangle=\operatorname{det}\left[\boldsymbol{S}_{(k-)\left(k^{\prime}-\right)}\right] .
$$

If we define the "hopping" elements $t_{p, q}$ through

$$
t_{k, k-q}:=\left[v^{\dagger}(k) v(k-q)\right]_{--},
$$

the matrix $\boldsymbol{S}_{(k-)\left(k^{\prime}-\right)}$ in Eq. (3.12) can be represented by a tight-binding Hamiltonian as

$$
\mathcal{S}=\sum_{k, k^{\prime}} a_{k}^{\dagger} \boldsymbol{S}_{(k-)\left(k^{\prime}-\right)} a_{k^{\prime}}=\sum_{k} \sum_{q} f_{q} t_{k, k-q} a_{k}^{\dagger} a_{k-q},
$$

where $a_{p}^{\dagger}\left(a_{p}\right)$ represents a fermionic creation (annihilation) operator defined for $p \in \mathrm{BZ}$. This Hamiltonian can be interpreted as describing a quantum particle hopping on a 1D lattice. Note that the gauge field $A_{x}(k)$ and the metric $g_{x x}(k)$ are related to the phase and the amplitude of the nearestneighbor hopping elements $t_{k, k-2 \pi / N}$, respectively. The hopping matrix $t_{k, k-q}$ is generically nonlocal. Also, since the kink operator introduces a sharp boundary in the real space, the dual Hamiltonian is highly nonlocal in $k$ space.

It is evident from Eq. (3.12) that the vanishing of $\langle\Psi|\eta| \Psi\rangle$ is equivalent to the existence of zero modes in the

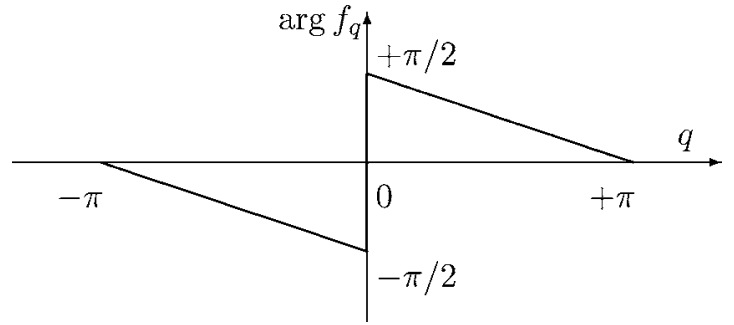
$N-1$.

FIG. 3. $\arg f_{q}=\arg \left(4 \frac{1}{1-e^{-\mathrm{i} q}}\right)=-\arg \left(1-e^{-\mathrm{i} q}\right)$ for $n_{q}=1,3,5, \ldots$,

spectrum of the $\mathcal{S}$ Hamiltonian. As we will see below, the spectrum of the $\mathcal{S}$ Hamiltonian is pretty much similar to that of the $\mathcal{C}$ Hamiltonian: away from a critical point, the spectrum is gapped and all the eigenvalues are close to either +1 or -1 , except a few eigenvalues in the gap that reflect the Berry phase if it is nontrivial. If the Berry phase is $\pi$ $\times$ (odd integer), there are exact zero-energy eigenmodes. When we approach a critical point, eigenvalues proliferate around zero-energy. Roughly speaking, the entanglement entropy takes into account the distribution of all the eigenvalues of $\mathcal{S}$, whereas the kink operator only takes into account the products of all the eigenvalues.

\section{B. "Chiral symmetry" and "time-reversal symmetry"}

The $\mathcal{S}$ Hamiltonian has a chiral symmetry. It directly reflects our bipartitioning the original system and has nothing to do with the chiral symmetry in the original system. Indeed, from Eq. (3.9), one can see that $a_{k}$ with $k$ odd/even are connected to $a_{k^{\prime}}$ with $k^{\prime}$ odd/even only. All the eigenstates in $k$ space are connected to their partner with the opposite energy via

$$
a_{k} \rightarrow a_{k}^{\prime}=(-1)^{i \pi n_{k}} a_{k}, \quad k=\frac{2 \pi n_{k}}{N}
$$

which in turn means in the real space

$$
a_{x} \rightarrow a_{x+N_{A}}=a_{x}^{\prime} .
$$

When the original system respects the chiral symmetry (not to be confused with the chiral symmetry above), all the single-particle wave function $\psi(k)$ of $\mathcal{S}$ in $k$ space can be taken to be real by a suitable rotation in $\boldsymbol{R}$ space. [However, when the Berry phase is $\gamma=\pi \times$ integer, this comes with requirement to have a Dirac string that intersects $\boldsymbol{R}(k)$.] The ability of taking all "hopping" elements $t_{k, k^{\prime}}$ to be real induces an additional "time-reversal symmetry" to $\mathcal{S}$ Hamiltonian; the phase associated with $f_{q}$ can be removed by a simple gauge transformation,

$$
a_{k} \rightarrow b_{k}=e^{+i k / 2-i k N_{A} / 2} a_{k} .
$$

(See Fig. 3.) Thus, we can take all the matrix elements $f_{q} t_{k, k-q}$ in the $\mathcal{S}$ Hamiltonian to be real. Furthermore, when we go back to the real space, this "time-reversal" invariance implies a parity symmetry with respect to an inversion center $x_{0}=-N_{A} / 2+1 / 2$. To see this, we first note that all the oneparticle eigenstates of $\mathcal{S}$ can be taken real in the basis 
$\left\{b_{p}^{\dagger}, b_{p}\right\}$; the $\mathcal{S}$ Hamiltonian can be diagonalized as $\mathcal{S}$ $=\sum_{n} \epsilon_{n} d_{n}^{\dagger} d_{n}$ with

$$
b_{p}=\sum_{n} \phi_{n}(p) d_{n}, \quad b_{p}^{\dagger}=\sum_{n} \phi_{n}(p) d_{n}^{\dagger},
$$

where $\phi_{n}(p)$ is an eigen wave function which is real. Since the basis $\left\{a_{x}^{\dagger}, a_{x}\right\}$ and $\left\{d_{n}^{\dagger}, d_{n}\right\}$ are related through

$$
a_{x}=\sum_{n} \frac{1}{\sqrt{N}} \sum_{k} e^{i k\left(x-1 / 2+N_{A} / 2\right)} \phi_{n}(k) d_{n},
$$

the real space eigen wave functions $\psi_{n}(x)$ in the basis $\left\{a_{x}^{\dagger}, a_{x}\right\}$ are given by

$$
\psi_{n}(x)=\frac{1}{\sqrt{N}} \sum_{k} e^{i k\left(x-1 / 2+N_{A} / 2\right)} \phi_{n}(k),
$$

from which one can see $\psi_{n}(x)$ satisfies

$$
\left[\psi_{n}(x)\right]^{*}=\psi_{n}\left(-x+1-N_{A}\right) \text {. }
$$

That is, the wave-function amplitude is parity symmetric with respect to $x_{0}=-N_{A} / 2+1 / 2$.

This time-reversal symmetry, which plays an important role for the vanishing of the expectation value of the kink operator. Indeed, it is this symmetry that guarantees the existence of zero modes of $\mathcal{S}$.

\section{Existence of zero modes}

The argument that tells us of the existence of zero modes for the $\mathcal{S}$ Hamiltonian is somewhat similar to the "proof" of the existence of zero modes for the $\mathcal{C}$ Hamiltonian in that we consider an adiabatic change of the Hamiltonian. The major difference comes from the fact that the chiral symmetry in the $\mathcal{S}$ Hamiltonian is implemented as a kind of time-reversal symmetry, as we discussed before.

We first establish that there is a pair of zero modes for $\mathcal{S}$ when we take $|\Psi\rangle$ as the ground state of the dimerized Hamiltonian (2.10) with the chiral symmetry. The hopping elements in $\mathcal{S}$ are computed from the overlap of the Bloch wave functions as

$$
\left\langle v_{ \pm}(p) \mid v_{ \pm}(q)\right\rangle=\frac{1}{2 R\left(R \mp R^{3}\right)}\left[\Delta^{2} e^{i(p-q)}+R^{2} \mp 2 R \xi+\xi^{2}\right] .
$$

The $\mathcal{S}$ Hamiltonian is then diagonalized as

$$
\mathcal{S}=\frac{1}{2 R\left(R-R^{3}\right)} \sum_{x}\left[\Delta^{2} f(x+1)+(R-\xi)^{2} f(x)\right] a_{x}^{\dagger} a_{x} .
$$

We see that there are two midgap states with energies $\pm \xi / R$. Especially when $\xi=0$, there are a pair of zero-energy states localized at the interfaces.

We then change the Hamiltonian in a continuous fashion in such a way that (i) it respects the chiral symmetry during the deformation, and (ii) it does not cross the gap closing point (the origin of $\boldsymbol{R}$ space). During this deformation, the Berry phase of the ground-state wave function is always kept to be $\pi$. As already discussed, we can take all the Bloch wave functions to be real and there is a "time-reversal" symmetry.

One can see that the zero modes never escape from $E$ $=0$ as it is constrained by the time-reversal symmetry, which is nothing but the parity invariance with respect to $x_{0}=$ $-N_{A} / 2+1 / 2$. First note that since the $\mathcal{S}$ Hamiltonian in $k$ space is nonlocal, it is short-ranged (quasidiagonal) in the real space. Thus, if we take the thermodynamic limit $N$ $\rightarrow \infty$, states that appear between the gap are spatially localized near the interfaces located $x=1 / 2$ and $x=N_{A}+1 / 2$, which separate the system into the two subsystems.

During the deformation, the two localized states, which are located at $x=1 / 2$ and $x=N_{A}+1 / 2$, respectively, can in principle go away from $E=0$. Due to the "chiral symmetry" of the $\mathcal{S}$ Hamiltonian, if one goes up from $E=0$, the other must go down. However, if there is "time-reversal symmetry," each eigenstate must be invariant under the space inversion with respect to $-N_{A} / 2+1 / 2$. In order for the localized states to satisfy these two conditions, both of them must be located at $E=0$.

As an example, the spectrum of the $\mathcal{S}$ Hamiltonian for the SSH model is presented in Fig. 2(c). The spectrum is almost identical to that of the $\mathcal{C}$ Hamiltonian, and a pair of zero modes persists for the entire quantum phase $\phi \in[-1,0)$.

\section{2D SYSTEMS WITH THE NONVANISHING CHERN NUMBER}

As far as we consider translational invariant systems, the above 1D discussions still apply to higher dimensions. When a $d$-dimensional translational invariant system is bipartitioned by a $(d-1)$-dimensional hyperplane, we can perform the $(d-1)$-dimensional Fourier transformation along the interface. The Hamiltonian is block-diagonal in terms of the wave number along the interface $\boldsymbol{k}_{\|}, \mathcal{H}:=\Sigma_{\boldsymbol{k}_{\|}} \mathcal{H}\left(\boldsymbol{k}_{\|}\right)$, where $\mathcal{H}\left(\boldsymbol{k}_{\|}\right)$is a 1D Hamiltonian for each $\boldsymbol{k}_{\|}$subspace. Then, the previous discussion applies to each $\mathcal{H}\left(\boldsymbol{k}_{\|}\right)$. As an example of a 2D two-band system, let us consider a $2 \mathrm{D}$ chiral $p$-wave superconductor ( $p$-wave SC) defined by

$$
\begin{aligned}
\mathcal{H}= & \sum_{\boldsymbol{r}} \boldsymbol{c}_{\boldsymbol{r}}^{\dagger}\left(\begin{array}{cc}
t & \Delta \\
-\Delta & -t
\end{array}\right) \boldsymbol{c}_{\boldsymbol{r}+\hat{x}}+\text { H.c. }+\boldsymbol{c}_{r}^{\dagger}\left(\begin{array}{cc}
t & i \Delta \\
i \Delta & -t
\end{array}\right) \boldsymbol{c}_{r+\hat{y}}+\text { H.c. } \\
& +\boldsymbol{c}_{\boldsymbol{r}}^{\dagger}\left(\begin{array}{cc}
\mu & 0 \\
0 & -\mu
\end{array}\right) \boldsymbol{c}_{\boldsymbol{r}},
\end{aligned}
$$

where the integral index $\boldsymbol{r}$ runs over the 2D square lattice, $\hat{\boldsymbol{x}}=(1,0), \hat{\boldsymbol{y}}=(0,1)$, and $t, \Delta, \mu \in \mathbb{R}$. For simplicity, we set $t$ $=\Delta=1$ in the following. The chiral $p$-wave SC has been discussed in the context of superconductivity in a ruthenate and paired states in the fractional quantum Hall effect. ${ }^{28-33}$ There are four phases separated by three quantum critical points at $\mu=0, \pm 4$, which are labeled by the Chern number $\mathrm{Ch}$ as $\mathrm{Ch}=0(|\mu|>4), \mathrm{Ch}=-1(-4<\mu<0)$, and $\mathrm{Ch}=+1(0<\mu<$ $+4)$. The nonzero Chern number implies the IQHE in the spin transport. ${ }^{30}$

The energy spectrum of the family of Hamiltonians $\mathcal{H}\left(k_{y}\right)$ parametrized by the wave number in the $y$ direction, $k_{y}$, is 

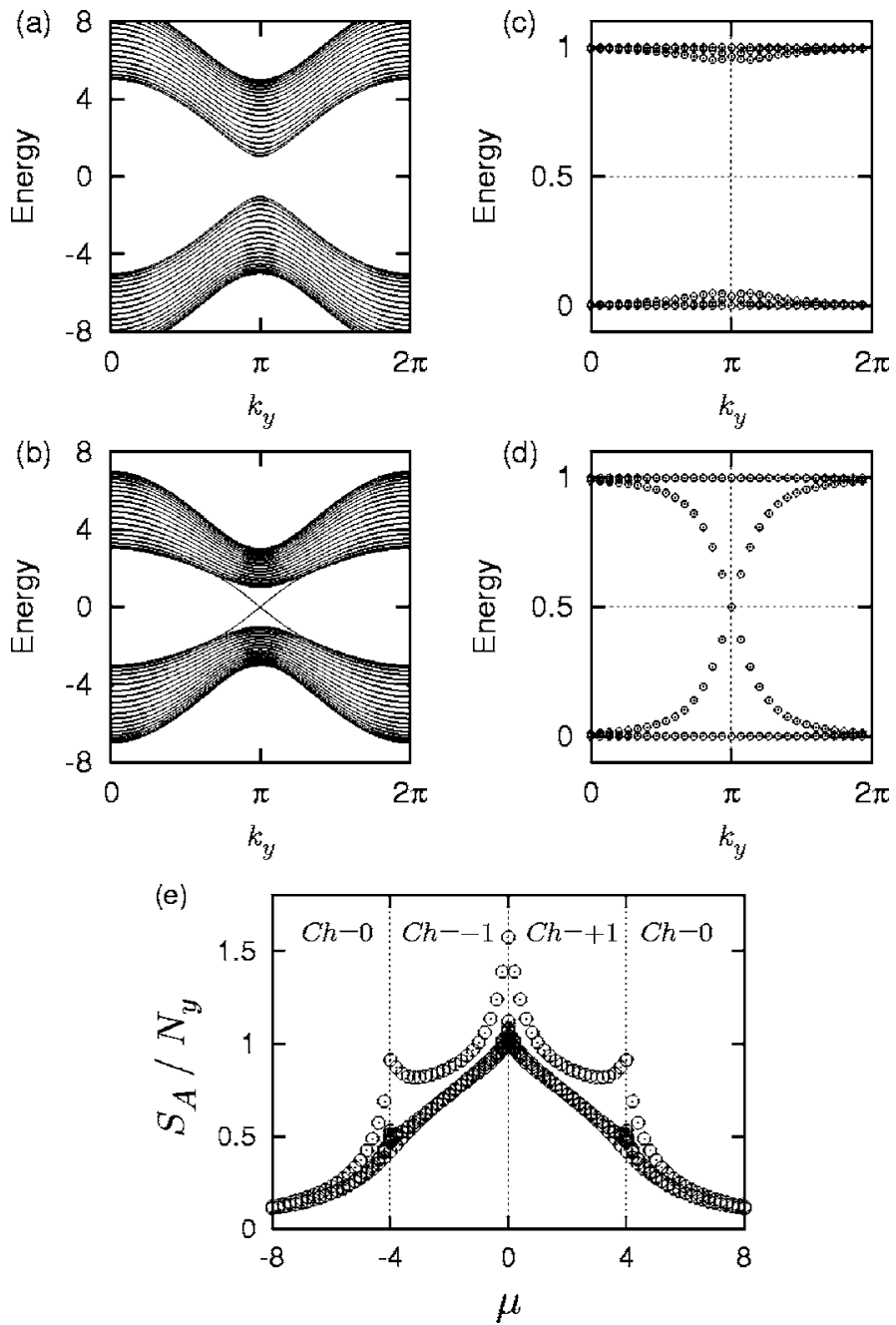

FIG. 4. The energy spectrum (measured in units of the hopping $t=1)$ vs $k_{y} \in[0,2 \pi)$ for the $2 \mathrm{D} p$-wave $\mathrm{SC}$ with boundaries. The chemical potential is $\mu=-5$ (a) and -3 (b) and $t=\Delta=1$. The corresponding spectra of the $\mathcal{C}$ Hamiltonian are shown in (c) $(\mu=-5)$ and (d) $(\mu=-3)$. The entanglement entropy of the $2 \mathrm{D}$ chiral $p$-wave SC as a function of $\mu$ is presented in (e). The aspect ratio $r=N_{y} / N_{x}$ is $r=1 / 2,1 / 3,1 / 4,1 / 8$ from the bottom at $\mu=-4$.

given in Figs. 4(a) and 4(b). There are branches of edge states that connect the upper and lower band for phases with $\mathrm{Ch}= \pm 1$. These edge states contribute to the entanglement entropy. The energy spectrum of the $\mathcal{C}$ Hamiltonian with open ends is shown in Figs. 4(c) and 4(d). The corresponding entanglement entropy is also found in Fig. 4(d) for several values of the aspect ratio $r=N_{y} / N_{x}$. We can see that for small $r$, the entanglement entropy shows a cusplike behavior at quantum phase transitions, whereas for larger values of $r$, the cusp is less eminent.

This behavior can be understood as a dimensional crossover of the scaling behavior of the entanglement entropy between 1D and 2D. For small $r$, the entropy behaves 1Dlike and the cusp is reminiscent of the logarithmic divergent behavior $S_{A} \sim \ln N_{A}$ of the pure 1D case. ${ }^{3}$ On the other hand, for $r$ close to unity, the entropy exhibits 2D behavior. In the pure 2D limit $(r=1)$, noting that the band structure at the critical points $\mu= \pm 4$ consists of one gapless Dirac fermion, the entanglement entropy scales as $S_{A}=\alpha N_{y}-\beta N_{y} / N_{A}$, where $\alpha, \beta$ is some constant (see Appendix B). Notice that unlike the case of a finite Fermi surface, ${ }^{34,35} S_{A} / N_{y}$ is constant for a Dirac fermion.

An interesting and direct application of the present section is the entanglement entropy of $2 \mathrm{D} d$-wave superconductors and carbon nanotubes. In these systems, different ways of bipartitioning the system lead to different amounts of the entanglement entropy. ${ }^{17}$

\section{CONCLUSION}

In this paper, we have identified two types of contributions to the entanglement entropy, i.e., one from the boundaries of the system created by taking the partial trace and the other from the bulk energy spectrum. The contribution from the boundaries is controlled by the Berry phase and hence we can make use of some known facts on the "bulk-boundary correspondence" to compute the entanglement entropy. Especially, we have obtained the lower bound of the entanglement entropy for 1D systems with discrete particle-hole symmetries. Intuitively, this means that when the Berry phase is zero, the ground-state wave function is very close to a simple product state, and there is not much entanglement. Thus, ground states with nontrivial Berry phase can be said to be more entangled in general.

Recently, it has been revealed that the Berry phase manifests itself in the semiclassical equation of motion, ${ }^{36}$ the density of states, ${ }^{37}$ and the anomalous Hall effect, etc. One can put the Berry phase correction to the entanglement entropy in the catalog.

One of the main messages of this paper is the superiority of the entanglement entropy to conventional correlation functions of local operators to describe quantum phases. Indeed, we clarified that the entanglement entropy is related to nonlocal operators, namely the twist operator and kink operator. The bulk contribution to the entanglement entropy is related to the localization length (correlation length), which is the real part of the logarithm of the expectation value of the twist operator and can be expressed by the quantum metric. ${ }^{23}$ On the other hand, the edge contribution is tied with the imaginary part and to the Berry phase [see Eqs. (2.4)-(2.7)]. We have also made a connection between the entanglement entropy and the kink operator. It is known that several phases of 1D strong correlated systems (such as the Haldane phase) can be described by these nonlocal operators. Another connection of the entanglement entropy to some sort of nonlocal operator can also be seen in a recent proposal of a holographic derivation of the entanglement entropy. ${ }^{38}$

Thus, the entanglement entropy can potentially be very useful to detect several quantum phases that need a more subtle way of characterization than classically ordered phases. For example, the entanglement entropy can be applied to several types of spin liquid ground states, which are speculated to be described by some kind of gauge theories. Indeed, for gapped phases of topological orders, this direction has already been explored to some extent. ${ }^{39,40}$

However, in order to push this direction further, we still need to deepen our understanding of the entanglement en- 
tropy. For example, extensions to multiband systems, especially to the case of completely degenerate bands, might also be interesting; we need to use the non-Abelian Berry phase to characterize the system. ${ }^{41}$ It is also interesting to investigate if the Berry phase of quantum ground states can be captured by other types of entanglement measures, such as the concurrence ${ }^{42}$ Finally, among many other questions, we need to consider how we can measure the entanglement entropy in a direct fashion. ${ }^{43}$

\section{ACKNOWLEDGMENTS}

We are grateful to R. Shindou and T. Takayanagi for useful discussions. This research was supported in part by the National Science Foundation under Grant No. PHY99-07949 (S.R.) and a Grant-in-Aid from the Ministry of Education, Culture, Sports, Science and Technology, Japan (Y.H.).

\section{APPENDIX A: THE DUAL BERRY PHASE}

In this appendix, we introduce the dual Berry phase $\bar{\gamma}$ in the 1D two-band Hamiltonian Eq. (2.1). If we impose the chiral symmetry, in a quantum phase with $\gamma=0$ the dual Berry phase is given by $\bar{\gamma}=-\pi$ whereas when $\gamma=-\pi, \bar{\gamma}=0$. Thus a quantum phase is characterized by both $\gamma$ and $\bar{\gamma}$.

It is in spirit similar to the dual order parameter (disorder parameter) in the quantum Ising spin chain. The 1D quantum Ising model in a transverse field has two phases: ordered and disordered. It is known that the entanglement entropy is $S_{A}$ $\geqslant 2 \ln 2$ for the former and $S_{A} \geqslant 0$ for the latter. On the other hand, they are related to each other by the Kramers-Wanier duality and hence one may argue that they are essentially equivalent. Why is the entanglement entropy in the ordered phase larger than that in the disordered phase? The reason is that Kramers-Wanier duality transformation is a nonlocal transformation and does not leave original bipartitioning invariant.

Similarly, the duality that we will introduce momentarily connects two different Hamiltonians with different Berry phase and entanglement entropy. It is possible since it is a transformation that changes the way of labeling the sites and hence bipartitioning.

Let us first introduce dimer operators by

$$
d_{ \pm, x+(1 / 2)}^{\dagger}=\frac{1}{\sqrt{2}}\left(c_{+, x}^{\dagger} \pm c_{-, x+1}^{\dagger}\right) .
$$

When written in terms of the dimer operators, the Hamiltonian (2.1) reads $\mathcal{H}=\frac{1}{2} \Sigma_{x, x^{\prime}}^{\mathrm{PBC}} \boldsymbol{d}_{x+(1 / 2)}^{\dagger} \bar{H}_{x-x^{\prime}} \boldsymbol{d}_{x^{\prime}+(1 / 2)}$, where the new hopping matrix elements $\bar{H}_{x-x^{\prime}}$ are some function of the original ones $H_{x-x^{\prime}}$. For simplicity, we focus on the case of particle-hole symmetric $\left(t_{+}=-t_{-}\right)$and translational invariant systems. In the momentum space, the Hamiltonian is given by $\mathcal{H}=\Sigma_{k \in \mathrm{BZ}} \boldsymbol{d}_{k}^{\dagger} \overline{\boldsymbol{R}}(k) \cdot \boldsymbol{\sigma} \boldsymbol{d}_{k}$ with a $3 \mathrm{D}$ vector $\overline{\boldsymbol{R}}(k)$ given by

$$
\bar{R}_{x}(k)=R_{z}(k),
$$

$$
\begin{aligned}
& \bar{R}_{y}(k)=\sin (k) R_{x}(k)+\cos (k) R_{y}(k), \\
& \bar{R}_{z}(k)=\cos (k) R_{x}(k)-\sin (k) R_{y}(k) .
\end{aligned}
$$

We define the dual Berry phase $\bar{\gamma}$ as the Berry phase for the dual 3D vector $\overline{\boldsymbol{R}}(k)$,

$$
\bar{\gamma}:=\int_{0}^{2 \pi} d k\left\langle\bar{v}(k)\left|\frac{d}{d k}\right| \bar{v}(k)\right\rangle=\int_{0}^{2 \pi} d k \frac{\bar{X} \bar{Y}-\bar{Y} \bar{X}}{2 \bar{R}(\bar{R}-\bar{Z})},
$$

where $\dot{X}=d R_{x}(k) / d k$, etc. Rotating $\overline{\boldsymbol{R}}$ around the $\bar{R}_{y}$ axis as $\left(\bar{R}_{x}, \bar{R}_{y}, \bar{R}_{z}\right) \rightarrow\left(\bar{R}_{z}, \bar{R}_{y},-\bar{R}_{x}\right)$, and noting $\bar{Y} \dot{\bar{X}}-\bar{X} \dot{\bar{Y}}=X \dot{Y}-Y \dot{X}$ $+X^{2}+Y^{2}$, the dual Berry phase is thus given by

$$
\bar{\gamma}=-\gamma-\pi-\int_{0}^{2 \pi} d k \frac{Z}{2 R} .
$$

Especially if we impose the chiral symmetry, $\boldsymbol{R}$ is restricted to the $X Y$ plane and thus

$$
\bar{\gamma}=-\gamma-\pi .
$$

\section{APPENDIX B: ENTANGLEMENT ENTROPY FOR A DIRAC FERMION IN 2D}

In this appendix, we estimate how much entanglement entropy is carried by a gapless Dirac fermion. It is an interesting question since a Dirac fermion is just in between a fully gapped system and a system with a finite Fermi surface; for the former, the entanglement entropy satisfies the area law, whereas for the latter there is a log-correction to the area law. ${ }^{34,35}$

Unlike the case of a finite Fermi surface, the entanglement entropy divided by $N_{y}, S_{A} / N_{y}$, is constant for a system with Dirac fermions, as can be seen as follows. The energy spectrum close to a gap closing point in the BZ, $\boldsymbol{k}^{(0)}$, is linear and so is the mass gap as a function of $k_{y}, m\left(k_{y}\right) \propto k_{y}-k_{y}^{(0)}$. The known result for a massive 1D system tells us each $k_{y}$ contributes to the entanglement entropy by $\sim \ln m\left(k_{y}\right)^{-1}$. If the length $N_{A}$ of the subsystem $A$ in the $x$ direction is finite, we expect the contributions from those $k_{y}$ with $m\left(k_{y}\right)^{-1} \geqslant N_{A}$ are given by $\sim \ln N_{A}$ instead. Then, the entanglement entropy can be evaluated by summing over the entanglement entropy for each 1D system with a fixed $k_{y}$,

$$
\frac{S_{A}}{2}=\sum_{k_{y}>0}^{m\left(k_{y}\right)^{-1} \leqslant N_{A}} \frac{2}{6} \ln m\left(k_{y}\right)^{-1}+\sum_{k_{y}>0}^{m\left(k_{y}\right)^{-1} \geqslant N_{A}} \frac{1}{3} \ln N_{A} .
$$

Converting the summation to the integral, we see that the entanglement entropy behaves as $S_{A}=\alpha N_{y}-\beta N_{y} / N_{A}$ for a single Dirac fermion where $\alpha, \beta$ is some constant. Hence $S_{A} / N_{y}$ is finite. This crude approximation is actually overestimating the entropy, but it is enough to derive essential features of the entropy. For more detailed analysis using the entropic $c$-function, see Ref. 44. 
${ }^{1}$ X. G. Wen, Phys. Rev. B 40, R7387 (1989); X. G. Wen and Q. Niu, ibid. 41, 9377 (1990); X. G. Wen, Quantum Field Theory of Many-Body Systems (Oxford University Press, New York, 2004).

${ }^{2}$ The base of the logarithm can be taken either $e$ or 2, depending on the literature.

${ }^{3}$ C. Holzhey, F. Larsen, and F. Wilczek, Nucl. Phys. B 424, 443 (1994).

${ }^{4}$ A. Osterloh, L. Amico, G. Falci, and R. Fazio, Nature (London) 416, 608 (2002).

${ }^{5}$ T. J. Osborne and M. A. Nielsen, Phys. Rev. A 66, 032110 (2002).

${ }^{6}$ G. Vidal, J. I. Latorre, E. Rico, and A. Kitaev, Phys. Rev. Lett. 90, 227902 (2003).

${ }^{7}$ H. Fan, V. Korepin, and V. Roychowdhury, Phys. Rev. Lett. 93, 227203 (2004).

${ }^{8}$ P. Calabrese and J. Cardy, J. Stat. Mech.: Theory Exp. 2004, 002 (2004).

${ }^{9}$ S. Furukawa, G. Misguich, and M. Oshikawa, Phys. Rev. Lett. 96, 047211 (2006).

${ }^{10}$ M. V. Berry, Proc. R. Soc. London, Ser. A A392, 45 (1984).

${ }^{11}$ D. J. Thouless, M. Kohmoto, M. P. Nightingale, and M. den Nijs, Phys. Rev. Lett. 49, 405 (1982).

${ }^{12}$ M. Kohmoto, Ann. Phys. (N.Y.) 160, 355 (1985).

${ }^{13}$ R. D. King-Smith and D. Vanderbilt, Phys. Rev. B 47, R1651 (1993); 48, 4442 (1993).

${ }^{14}$ R. Resta, Rev. Mod. Phys. 66, 899 (1994).

${ }^{15}$ M. den Nijs and K. Rommelse, Phys. Rev. B 40, 4709 (1989).

${ }^{16}$ M. Nakamura and S. Todo, Phys. Rev. Lett. 89, 077204 (2002).

${ }^{17}$ S. Ryu and Y. Hatsugai, Phys. Rev. Lett. 89, 077002 (2002).

${ }^{18}$ Y. Hatsugai, Phys. Rev. Lett. 71, 3697 (1993).

${ }^{19}$ A. Y. Kitaev, Proceedings of the Mesoscopic and Strongly Correlated Electron Systems Conference (July, 2000, Chernogolovka, Moscow Region, Russia), cond-mat/0010440 (unpublished).
${ }^{20}$ J. Zak, Phys. Rev. Lett. 62, 2747 (1989).

${ }^{21}$ S. Onoda, S. Murakami, and N. Nagaosa, Phys. Rev. Lett. 93, 167602 (2004).

${ }^{22}$ The expectation value of the twist operator [Eq. (2.5)] can be obtained in a similar fashion to that of the kink operator, see Sec. III A.

${ }^{23}$ N. Marzari and D. Vanderbilt, Phys. Rev. B 56, 12847 (1997).

${ }^{24}$ I. Peschel, J. Phys. A 36, L205 (2003).

${ }^{25}$ A similar criterion can be developed for systems with Kramers degeneracy. There, the time-reversal symmetry can be used in addition to the chiral symmetry. ${ }^{17}$

${ }^{26}$ A. Heeger, S. Kivelson, J. R. Schrieffer, and W.-P. Su, Rev. Mod. Phys. 60, 781 (1988).

${ }^{27}$ R. Shindou, K. Imura, and M. Ogata (unpublished).

${ }^{28}$ N. Read and D. Green, Phys. Rev. B 61, 10267 (2000).

${ }^{29}$ G. E. Volovik, JETP Lett. 66, 493 (1997).

${ }^{30}$ T. Senthil, M. P. A. Fisher, L. Balents, and C. Nayak, Phys. Rev. Lett. 81, 4704 (1998).

${ }^{31}$ J. Goryo and K. Ishikawa, Phys. Lett. A 260, 294 (1999).

${ }^{32}$ Y. Morita and Y. Hatsugai, Phys. Rev. B 62, 99 (2000).

${ }^{33}$ Y. Hatsugai and S. Ryu, Phys. Rev. B 65, 212510 (2002).

${ }^{34}$ M. M. Wolf, Phys. Rev. Lett. 96, 010404 (2006).

${ }^{35}$ D. Gioev and I. Klich, Phys. Rev. Lett. 96, 100503 (2006).

${ }^{36}$ G. Sundaram and Q. Niu, Phys. Rev. B 59, 14915 (1999).

${ }^{37}$ D. Xiao, J. Shi, and Q. Niu, Phys. Rev. Lett. 95, 137204 (2005).

${ }^{38} \mathrm{~S}$. Ryu and T. Takayanagi, hep-th/0603001 (unpublished).

${ }^{39}$ A. Kitaev and J. Preskill, Phys. Rev. Lett. 96, 110404 (2006).

${ }^{40}$ M. Levin and X. G. Wen, Phys. Rev. Lett. 96, 110405 (2006).

${ }^{41}$ Y. Hatsugai, J. Phys. Soc. Jpn. 73, 2604 (2004); 74, 1374 (2005).

${ }^{42}$ W. K. Wootters, Phys. Rev. Lett. 80, 2245 (1998).

${ }^{43}$ I. Klich, G. Refael, and A. Silva, cond-mat/0603004 (unpublished).

${ }^{44}$ H. Casini and M. Huerta, J. Stat. Mech.: Theory Exp. 2005, 012 (2005). 